\title{
La regulación jurídica de los residuos de construcción demolición (RCD) en España. El caso de la Comunidad de Madrid
}

\author{
Legal aspects about construction and demolition waste \\ (CDW) in Spain. The Autonomus Region of Madrid
}

M. del Río( ${ }^{(*)}$, P. Izquierdo ${ }^{(*)}$, I. Salto ${ }^{(*)}$, J. Santa Cruz $z^{(*)}$

\section{RESUMEN}

España es el quinto país europeo que genera más residuos de construcción-demolición (RCD), y uno de los que menos esfuerzos realiza para recuperarlos según el informe Symonds \& Ass. Este informe indica que el $90 \%$ de los 13 millones de toneladas de materiales inertes que se generan cada año, acaban en vertederos y sólo un $5 \%$ se recicla.

En nuestro país la competencia sobre la gestión de los RCD corresponde a las Comunidades Autónomas, a excepción de los RCD procedentes de obras menores domiciliares, por lo que éstas a la vista de este problema, han redactado Planes de gestión de RCD en base a los Planes Nacionales. Estos planes han sido redactados tomando como base el denominado principio de jerarquía, contemplado en el artículo 1.1 de la Ley 10/1998 de Residuos. Este principio establece como prioritario prevenir en la medida de lo posible, reutilizar lo que se pueda, reciclar lo que no se pueda reutilizar y valorizar energéticamente todo lo que no se pueda reutilizar o reciclar. El depósito final en vertedero es la última opción, la menos satisfactoria. En este artículo se presentan algunas de las medidas legales que la Comunidad de Madrid ha impulsado, en los últimos años, para la mejora de la gestión de los RCD en obras de edificación.

$113-90$

Palabras clave: gestión residuos; residuos de construcción y demolición (RCD); demolición; reciclaje; reutilización; sostenibilidad.
SUMMARY

Spain is the fifth country in Europe generating construction and demolition waste (CDW) and unfortunately, it is one which does the least to recuperate it, as stated in the Symonds \& Ass report. This report indicates that $90 \%$ of the 13 million tons of yearly generated waste materials end up in the dump and only 5\% is recycled.

In our country the management of CDW depends from the Autonomous Regions with the exception of the waste originated from minor house works; therefore, the Autonomous Regions have defined their management CDW plans referring to the National Plans. Those plans have been set based on the hierarchical principle, stated in title 1.1 of the 10/1998 Law referring to waste products. This principle establishes prevention as a top priority -whenever possible- followed by reutilization and recycling and in addition, to clearly assess all that cannot be reused o recycled. The final deposit in the dump is the last option, and the least satisfactory one. In this paper, some noticeable legal actions promoted in the last years by the Autonomous Region of Madrid to improve the management of CDW in buildings are presented.

Keywords: waste management; construction and demolition waste (CDW); demolition; recycled materials; materials reutilization; sustainable growth. 


\section{INTRODUCCIÓN}

Los Residuos de Construcción Demolición (RCD) se producen sobre todo durante la demolición, derribo, transformación o ampliación de edificaciones, infraestructuras, calles, caminos, aeropuertos, vías y demás superficies para el tráfico. Debido a su heterogeneidad, los que presentan una mayor dificultad para su reutilización o reciclaje son los residuos procedentes de edificaciones.

Estos residuos se consideran inertes, es decir, no peligrosos, que no experimentan transformaciones físicas, químicas o biológicas significativas.

En Europa, el catálogo Europeo de RCD (1), clasifica los residuos en:

- Mezclas de hormigón, ladrillos, tejas y materiales cerámicos.

- Madera, vidrio y plástico.

- Mezclas bituminosas, alquitrán de hulla y otros productos alquitranados.

- Metales (incluidas sus aleaciones).

- Tierra (incluida la excavada en zonas contaminadas), piedras y lodos de drenaje.

- Materiales de aislamiento y materiales de construcción que contienen amianto.

- Materiales de construcción a base de yeso.

- Otros residuos de construcción y demolición.

No obstante, dentro de cada grupo aparecen especificados algunos residuos como peligrosos, de conformidad con la Directiva 91/689/CEE sobre residuos peligrosos, a cuyas disposiciones están sujetos a menos que se aplique el apartado 5 del artículo 1 de esta Directiva.

\section{LA REUTILIZACIÓN Y EL RECICLADO DE LOS RCD EN ESPAÑA}

El importante incremento de la construcción que se ha venido produciendo en España, en la última década, así como la entrada en vigor de la nueva normativa del Ministerio de Fomento para la Inspección Técnica de Edificios, ha traído lógicamente como consecuencia un notable incremento en la generación de residuos de construcción y demolición (RCD), planteándose la urgente necesidad de habilitar mecanismos de gestión de esos residuos, capaces de dar respuesta a la situación creada.

Los residuos de construcción-demolición tienen un potencial de valorización recono- cido, pero éste está limitado por motivos técnicos (2), por razones de mercado y fundamentalmente por la falta de normativa de regulación de los mismos así como por los costes ridículos de su eliminación. Todo ello supone que en la actualidad reciclar sea más gravoso, en términos económicos, que eliminar.

Además, en la valorización de los RCD hay que tener en consideración cuestiones que pueden afectar al medioambiente, influyendo por tanto en la decisión final de reutilizar, reciclar o eliminar. Entre estas cuestiones destacan:

- La necesidad de transporte del residuo, ya que éste produce, contaminación del aire, ruido y vibraciones, y lógicamente consumo de energía.

- La recuperación de los RCD que en ocasiones genera polvo, ruido, vibraciones, aguas residuales, distorsiones en el entorno socioeconómico, e incluso, la recuperación de ciertos residuos peligrosos, como el amianto, puede producir impactos negativos sobre la salud de las personas.

Atendiendo a esto, la eliminación de los residuos puede ser positiva si se hace como un vertido controlado posibilitando la recuperación de zonas degradadas o como material de cubierta en vertederos, suponiendo en estos casos un ahorro en el consumo de materias primas, la preservación de Espacios Naturales e incluso la reducción de canteras.

Pero, en muchas otras ocasiones, la eliminación de residuos puede ser negativa si se realiza de forma incontrolada, dando lugar a puntos negros o modificando zonas de alto valor ecológico. Incluso puede acabar generando problemas de inestabilidad geológica con el tiempo.

Actualmente la media comunitaria sitúa el reciclaje de RCD entre el 25 y el $30 \%$ de la producción, terminando entre un 70 y un $75 \%$ en vertedero (3).

Los mercados potenciales existentes en España para la venta de materiales de producto reciclado son:

- Bases y sub-bases para firmes de carreteras de segundo orden, urbanizaciones, polígonos industriales y otras áreas de expansión.

- Áridos o residuos de ladrillos para drenajes, rellenos de zanjas y firmes de caminos. 
-Áridos o residuos de ladrillos para lechos de patios y azoteas.

- Áridos para hormigones pobres.

Además, teniendo en cuenta la valorización de los distintos elementos pertenecientes al grupo de los residuos inertes, existe un mercado emergente en cuanto a:

\section{- Residuos de chatarra férrica \\ -Vidrio \\ - Materiales nobles de la construcción y carpintería (cuarterones, cercos, rejas, portones, ventanas, marcos, etc.) \\ -Piedra labrada, fundamentalmente granito y basalto (adoquines, bordillos, losas, jambas, dinteles, etc.).}

\section{REGULACIÓN JURÍDICA DE LOS RESIDUOS DE CONSTRUCCIÓN DEMOLICIÓN EN ESPAÑA}

Por lo que se refiere a la regulación jurídica relativa a este tipo de residuos, estuvo en el pasado sujeta a la Ley 42/1975 (4), sobre desechos y residuos sólidos urbanos. A tenor de lo establecido en esta disposición, la gestión de este tipo de escombros quedaba dentro del ámbito de competencia municipal. Esto dio lugar a una falta de responsabilidad y de control efectivo sobre la gestión de los residuos y supuso una proliferación de vertidos incontrolados e indiscriminados en lugares próximos a los núcleos de población con fácil acceso rodado (márgenes de carreteras y caminos, vías pecuarias, ríos, arroyos, etc.).

Hace casi una década y debido fundamentalmente al fuerte crecimiento de la industria de la construcción en España, se generó un incremento muy importante de los residuos de construcción y demolición, por lo que se redactó el I Plan Nacional de gestión de residuos de construcción y demolición (3). Este Plan, realizaba unas previsiones de generación de residuos de 42 millones de toneladas (aproximadamente 1 ton/hab/año), sin embargo, los datos reales demuestran que dicha previsión fue superada especialmente en algunas comunidades autónomas, pues la producción media de RCD por habitante y año, según los datos de 2005, fueron de 790 kilos, con un máximo de 1.664 kg/hab.año en Castilla-La Mancha y un mínimo de 145 kg/hab.año en la ciudad de Ceuta.

Por ello y ante esta situación, se redacta el II Plan Nacional de Residuos de Construcción y Demolición (5). Este Plan, propone una serie de medidas basándose en los datos de un estudio del Instituto para la Sostenibilidad de los Recursos (ISR), de diciembre de 2004, para el Ministerio de Medio Ambiente.
En dicho estudio se hace referencia a que aproximadamente 10 millones de toneladas anuales de RCD, sobre un total de 35 millones de toneladas que estimaba que se generaban en esa fecha, se eliminaban en vertederos de residuos inertes que se consideran adaptados a la normativa de vertederos vigente. Pero lo más relevante es que, tal y como afirma el ISR en el citado estudio, todavía hoy en España más del $60 \%$ de los RCD generados se eliminan de forma incontrolada en escombreras, agujeros o vaguadas o son desperdigados a precio nulo (en algunos casos a unos céntimos de euro por tonelada), llevando mezclados en su masa residuos de todo tipo, incluso algunos potencialmente peligrosos.

Pues bien, enmarcado en este Plan se redacta el Real Decreto 105/2008 (6), por el que se regula la producción y gestión de los RCD, que va más allá de la normativa europea y contempla la concreta aplicación del principio de responsabilidad del productor y el poseedor a estos residuos.

En este RD desarrollado con el consenso de los agentes económicos y sociales del sector de la construcción, las Comunidades Autónomas, los Ayuntamientos y el Consejo Asesor de Medio Ambiente, describe que el régimen de control de la producción, posesión y gestión de los residuos de construcción y demolición se basa en la necesaria colaboración entre las comunidades autónomas y las entidades locales. No obstante, se contempla la posibilidad del establecimiento, en los términos previstos en la legislación de las comunidades autónomas, de un mecanismo de control vinculado a la obtención de la licencia de obras, mediante la constitución, por parte del productor de los residuos, de una fianza u otra garantía financiera equivalente, que responda del cumplimiento de los requisitos del RD y, en particular, de la gestión de los RCD que se producirán en la obra. Y aunque el RD adjudica el control de la producción, posesión y gestión de los residuos a las Comunidades Autónomas y a las entidades locales, distingue tres tipos de agentes implicados directamente en dicha gestión, y así mismo establece sus obligaciones: el productor de residuos, el poseedor de residuos y el gestor de residuos.

El Real Decreto se aplica a los RCD generados en obras de construcción, rehabilitación, reparación, reforma o demolición tanto de bienes inmuebles como de obra civil, quedando exentos: las tierras y piedras no contaminadas por sustancias peligrosas, los residuos de industrias extractivas, los lodos de dragado no peligrosos, etc., y las obras menores y reparaciones domiciliarias, que no precisen de proyecto. 
El objetivo fundamental del mismo es establecer el régimen jurídico de la producción y gestión de los RCD con el fin de fomentar su prevención, reutilización, reciclado y otras formas de valorización, y asegurar que los RCD destinados a operaciones de eliminación reciban un tratamiento adecuado para de esta manera contribuir a un desarrollo sostenible de la construcción, y evitando al mismo tiempo, entre otras cosas, la contaminación de suelos y acuíferos, el deterioro paisajístico y la eliminación de estos residuos sin aprovechamiento de sus recursos valorizables.

Para conseguir este objetivo el RD propone una serie de acciones entre las que se destacan las siguientes por su carácter innovador:

- Prohibir el depósito de RCD en vertedero sin tratamiento previo, exceptuando los residuos inertes cuyo tratamiento sea técnicamente inviable y los RCD cuyo tratamiento no contribuya a los objetivos establecidos en el RD ni a reducir los peligros para la salud humana o el medio ambiente.

- Obligar al productor (titular de la licencia) de los RCD, a la elaboración en el proyecto de obra, de un Estudio de Gestión de RCD para los residuos que se produzcan, así como una valoración del coste previsto para su gestión.

- Obligar al poseedor de los RCD (el que ejecuta la obra) a establecer la separación y clasificación de los residuos, elaborar un plan de ejecución y asumir los costes.

- Afianzar la posición empresarial de aqueIlos materiales reutilizables para los que ya existe un mercado (metal y madera) e incentiva la creación de otros mercados como el de los áridos reciclados.

- Propugnar una actitud ejemplarizante en aquellas obras en que las administraciones públicas intervengan como promotores. Además los órganos de contratación, al determinar los criterios de base para la valoración de ofertas tendrán en cuenta la consideración de medidas sobre prevención y reutilización o reciclado de los RCD, así como la utilización en las unidades de obra de áridos y otros productos procedentes de valorización de residuos.

- Fomentar medidas como los acuerdos voluntarios entre los responsables de la correcta gestión de los residuos y los responsables de la restauración de los espacios ambientalmente degradados, o con los titulares de obras de acondicionamiento o relleno para conseguir mejoras en la sostenibilidad de las obras.

Por tanto, en todo proyecto de obra, el productor de RCD debe incluir un Estudio de Gestión de RCD y previo a su ejecución, la dirección facultativa deberá aprobar el Plan de gestión de RCD, definido por el poseedor de RCD, en base al estudio de gestión.

\section{Estudio de Gestión de RCD}

El productor de los RCD está obligado a redactar o a encargar la redacción del Estudio de Gestión de RCD que deberá incluir, al menos:

- Una estimación de la cantidad, expresada en toneladas y en metros cúbicos, de los RCD que se generarán en la obra, codificados con arreglo a la lista europea, o norma que la sustituya.

- Las medidas para la prevención de residuos en la obra objeto del proyecto.

- Las operaciones de reutilización, valorización o eliminación a que se destinarán los residuos que se generarán en la obra.

- Las medidas para la separación de los residuos en obra, en particular, para el cumplimiento por parte del poseedor de los residuos.

- Los planos de las instalaciones previstas para el almacenamiento, manejo, separación y, en su caso, otras operaciones de gestión de los RCD dentro de la obra. Posteriormente, dichos planos podrán ser objeto de adaptación a las características particulares de la obra y sus sistemas de ejecución, previo acuerdo de la dirección facultativa de la obra.

- Las prescripciones del pliego de prescripciones técnicas particulares del proyecto, en relación con el almacenamiento, manejo, separación y, en su caso, otras operaciones de gestión de los residuos de construcción y demolición dentro de la obra.

- Una valoración del coste previsto de la gestión de los residuos de construcción y demolición que formará parte del presupuesto del proyecto en capítulo independiente. 


\section{Plan de Gestión de RCDs}

El Plan de Gestión de RCDs lo redactará el poseedor de los residuos y deberá aprobarse por parte de la dirección facultativa y además ser aceptado por la propiedad, pasando a formar parte de los documentos contractuales de la obra.

El plan deberá incluir:

- La descripción de cómo se aplicará el estudio de gestión del proyecto.

- La descripción de cómo se sufragará su coste.

- La documentación acreditativa de la correcta gestión de los residuos.

\section{GESTIÓN DE LOS RESIDUOS DE CONSTRUCCIÓN Y DEMOLICIÓN EN LA COMUNIDAD DE MADRID}

Como se ha comentado, en nuestro país la competencia sobre la gestión de los RCD corresponde a las Comunidades Autónomas, a excepción de los RCD procedentes de obras menores domiciliarias; a tal efecto, prácticamente la totalidad de las Comunidades Autónomas españolas han definido sus planes estratégicos para el tratamiento y la gestión de estos residuos.

En concreto, la Comunidad de Madrid, con una densidad 626 habitantes $/ \mathrm{km}^{2}$ y un importante auge del sector de la construcción durante los últimos años, ha necesitado implementar medidas paulatinas para minimizar el notable aumento de la generación de residuos de $C D$, siempre en colaboración con los Ayuntamientos. Estas medidas quedan definidas en el denominado "Plan de gestión integrada de los residuos de construcción y demolición de la comunidad de Madrid (2002-2011)" (7).

Los criterios o principios básicos del Plan de gestión son básicamente los siguientes:

- Principio de prevención en la generación de RCD en su lugar de origen y potenciación de los sistemas de separación y reciclaje, de aquellos materiales susceptibles de recuperación, mediante las operaciones de separación in situ y demolición selectiva.

- Establecimiento de la responsabilidad de los productores de residuos. Son éstos los que deben de prever y hacer frente a la responsabilidad de su correcta gestión ambiental, debiendo correr con los gastos de la misma.

- Principio de Priorización. Dado que no todos los métodos o técnicas para gestionar un residuo son igualmente satisfactorios desde el punto de vista ambiental y teniendo en cuenta la jerarquía que establece la Política Comunitaria de Residuos (prevención, reutilización, reciclado, valorización, vertido), se deberán favorecer aquellas actuaciones que primen la prevención y reutilización frente al vertido.

- Principio de proximidad. Se deben reducir en lo posible las distancias para el transporte de residuos, debiendo ser tratados en el centro más próximo posible a su lugar de origen o generación.

- Principio de internalización de costes, teniendo en cuenta todos los relativos al proceso de gestión de los residuos, para su correspondiente repercusión.

- Articulación de un sistema que obligue a los constructores y colegios profesionales a incluir en todos los proyectos, la adecuada gestión de RCD, incluyendo la financiación de dicha gestión.

El objetivo principal del Plan es la definición de un modelo de gestión que garantice el adecuado tratamiento de la totalidad de los RCD generados, así como resolver el déficit ambiental generado por la gestión de los RCD en años pasados. Para la consecución de este objetivo se están adoptando las siguientes medidas:

- Elaboración de la normativa necesaria para regularizar la producción y gestión de los RCD, desde la recogida hasta su valorización o eliminación.

- Creación y explotación de una red de instalaciones de gestión de RCD, que garantice el correcto tratamiento de la totalidad de los producidos.

- Elaboración de un sistema de tasas, cánones o precios que desincentiven el vertido.

- Diseño de un Programa de Sellado y Restauración de vertederos municipales de escombros no adaptables a las exigencias del Real Decreto 1481/2001 (8). 
- Desarrollo de programas de divulgación y concienciación ciudadana.

- Creación de una red de Puntos Limpios de Residuos de Construcción y Demolición, destinados exclusivamente a recibir los residuos generados en obras sometidas al procedimiento de acto comunicado.

- Redacción de una norma técnica de calidades de los materiales reutilizables o reciclables procedentes de los RCD.

\section{CONCLUSIONES}

En España, a pesar del aumento en la concienciación de la sociedad sobre la protección al medio ambiente y la necesidad del reciclaje, y a pesar de las iniciativas y la regulación jurídica llevada a cabo para promover estos principios, los RCD todavía encuentran una vía de escape fácil en los vertederos. Esto es debido, fundamentalmente a las bajas tasas de vertido, una solución claramente no soportable en el futuro, dado el incontrolado y constante volumen creciente de los vertidos, y la antagónica desaparición de los recursos naturales.

La legislación vigente de ámbito estatal y autonómico, está proponiendo, a través de los diversos Planes de Gestión de RCD, medidas para la disminución y gestión de estos residuos. En este sentido, es importante insistir en la necesidad fundamental de la coordinación entre las distintas Administraciones y las empresas del sector, de cara al intercambio de investigaciones y experiencias.

La Comunidad de Madrid, a través de su Plan de Gestión integrada de los residuos de construcción y demolición (2002-2011), también ha propuesto una serie de medidas como: la organización de una red pública de instalaciones (depósitos y plantas de reciclaje), un sistema de tasas y fianzas para los poseedores del residuo, programas de divulgación y concienciación ciudadana y un programa de sellado y restauración de vertederos municipales. El Plan, cuyo periodo de vigencia se extiende hasta el año 2011, trata de solventar la situación producida ante el notable aumento de la generación de residuos de construcción y demolición y la proliferación de escombreras ilegales y puntos negros de vertido. Aunque, en un principio, la Comunidad de Madrid está llevando a cabo las medidas propuestas, todavía queda mucho por hacer y al igual que ha ocurrido con los resultados del I Plan Nacional, se tendrá que esperar al año 2011 para determinar la validez de las mismas y para conocer la inversión real del desarrollo del Plan entre los años 20022011 que en un principio ascendía a 1,3 millones de euros.

\section{REFERENCIAS}

(1) Orden MAM/304/2002, de 8 de Febrero, por la que se publican las operaciones de valorización y eliminación de residuos y la lista europea de residuos. BOE 43, de 19-02-02

(2) Catálogo de residuos utilizables en la construcción (Cedex). Julio 2001.

Demolición y reutilización de estructuras de hormigón. Recomendaciones y Manuales Técnicos Estructuras y edificación (E-7). Colegio de Ingenieros de Caminos y Puertos de Madrid.

Hannecuart, Jean Pierre. "Guía de Buenas Prácticas para el reciclaje de los residuos plásticos. Una guía por y para las autoridades locales y regionales". ACRR 2004.

I Conferencia Nacional sobre Prevención de Residuos. Madrid. 5 y 6 de Abril de 2006. Ministerio de Medio Ambiente.

Manual de directrices para el uso de áridos reciclados en obras públicas de la Comunidad Autónoma del País Vasco. Gobierno Vasco 2009.

Floriach Puig, T. "La problemática de la gestión de los residuos de construcción, una aproximación al estado actual de la cuestión". BIA. Colegio Oficial de Aparejadores y Arq. Técnicos de Madrid. 2008.

(3) I Plan Nacional de Residuos de Construcción y Demolición (2001-2006). Resolución de 14 de junio de 2001, de la Secretaría General de Medio Ambiente, por la que se dispone la publicación del Acuerdo de Consejo de Ministros, de 1 de junio de 2001

(4) Ley 42/1975. 19 de noviembre sobre Desechos y Residuos Sólidos Urbanos. BOE 280, 21-11-75.

(5) Plan Nacional Integrado de Residuos para el período 2008-2015. II Plan Nacional de Residuos de Construcción y Demolición (2007-2015). Resolución de 20 de enero de 2009, de la Secretaría de Estado de Cambio Climático.

(6) Real Decreto 105/2008, de 1 de febrero, por el que se regula la producción y gestión de los residuos de construcción y demolición. BOE 38.

(7) Plan de gestión de RCD. Comunidad autónoma de Madrid. (2002-2011).

(8) Real Decreto 1481/2001, de 27 de diciembre, por el que se regula la eliminación de residuos mediante depósito en vertedero. BOE: 25 de 29/1/2002. 